\title{
Corylifol A from Psoralea corylifolia L. Enhances Myogenesis and Alleviates Muscle Atrophy
}

\author{
Yeongeun Han ${ }^{\dagger}$, Hyejin Lee ${ }^{\dagger}$, Hua Li and Jae-Ha Ryu *(D) \\ Research Institute of Pharmaceutical Sciences and College of Pharmacy, Sookmyung Women's University, 100 \\ Chungparo 47-Gil, Yongsan-Gu, Seoul 04310, Korea; thddl1428@naver.com (Y.H.); u9698115@naver.com (H.L.); \\ cooldog227@hotmail.com (H.L.) \\ * Correspondence: ryuha@sookmyung.ac.kr; Tel.: +82-2-710-9568 \\ + These authors contributed equally to this work.
}

Received: 23 January 2020; Accepted: 20 February 2020; Published: 25 February 2020

\begin{abstract}
Inflammatory conditions caused by cancer, chronic diseases or aging can lead to skeletal muscle atrophy. We identified myogenic compounds from Psoralea corylifolia (PC), a medicinal plant that has been used for the treatment of inflammatory and skin diseases. C2C12 mouse skeletal myoblasts were differentiated in the presence of eight compounds isolated from PC to evaluate their myogenic potential. Among them, corylifol A showed the strongest transactivation of $\mathrm{MyoD}$ and increased expression of myogenic markers, such as $\mathrm{MyoD}$, myogenin and myosin heavy chain (MHC). Corylifol A increased the number of multinucleated and MHC-expressing myotubes. We also found that the p38 MAPK signaling pathway is essential for the myogenic action of corylifol A. Atrophic condition was induced by treatment with dexamethasone. Corylifol A protected against dexamethasone-induced myotube loss by increasing the proportion of multinucleated MHC-expressing myotubes compared with dexamethasone-damaged myotubes. Corylifol A reduced the expression of muscle-specific ubiquitin-E3 ligases (MAFbx and MuRF1) and myostatin, while activating Akt. These dual effects of corylifol A, inhibition of catabolic and activation of anabolic pathways, protect myotubes against dexamethasone damage. In summary, corylifol A isolated from P. corylifolia alleviates muscle atrophic condition through activating myoblast differentiation and suppressing muscle degradation in atrophic conditions.
\end{abstract}

Keywords: Psoralea corylifolia; corylifol A; myogenesis; muscle atrophy; C2C12 myoblast

\section{Introduction}

A number of internal and external stimuli, including physical injury or inflammatory environments within the body, can induce skeletal muscle atrophy. An increase in the aging population is known to be a primary cause for the increased incidence of skeletal muscle atrophy (referred to as sarcopenia). Aging increases the risk of chronic diseases, including diabetes, obesity, abnormal endocrine regulation, depletion of hormones and cancer. These chronic diseases also cause muscle atrophy (referred to as cachexia). Thus, muscle atrophy is one of the most important diseases that need to be addressed in our increasingly aged society.

In cachexia and sarcopenia, reductions in the size and strength of muscles makes it difficult to control body movement and results in low quality of life as well as higher mortality [1-4]. To protect against atrophy, muscles regenerate through the processes of satellite cell activation, myogenesis and muscle protein synthesis. Quiescent satellite cells located beneath the basal lamina can be activated by physical injury or growth stimuli to regenerate muscles [5]. Activated muscle satellite cells proliferate, and myoblasts then express various myogenic factors to initiate myoblast differentiation. The fully differentiated myoblasts, i.e., myotubes, then fuse together to form muscle 
bundles. Therefore, activators of normally quiescent satellite cells have attracted attention as targets for developing muscle-strengthening drugs or supplements.

In addition to muscle protein synthesis, muscle degradation is also part of the homeostatic mechanism for maintaining muscle mass and fiber size. In muscle atrophic conditions, protein degradation pathways are upregulated through proteolytic machinery such as ubiquitin-proteasome, caspase, calpain, cathepsin L or autophagy. Potential therapeutic agents and several molecular mechanisms for regulating skeletal muscle atrophy have been recently reviewed [6]. At present, megestrol acetate is the only remedy approved by U.S. FDA for the treatment of AIDS- or cancer-induced cachexia. However, its side effects have increased the need for new drug developments [6]. In 2016, the CDC (Centers for Disease Control and Prevention) newly established an ICD-10-CM code for sarcopenia as an independent medical condition accompanying the age-associated losses of muscle mass, strength and function. At present, there are no FDA-approved drugs for sarcopenia. Considering the reliability and stability of medicinal plants that have long been in use, their application as therapeutic agents for muscle atrophy has been suggested [7]. Indeed, we have reported on several myogenic compounds from medicinal plants that can be used to develop anti-myopathy drugs [8-11].

The seeds of Psoralea corylifolia L. (Fabaceae, PC) have been traditionally used for the treatment of several symptoms, including stomachache, diuretic, leprosy, skin diseases such as eczema, leukoderma and psoriasis, in Asian countries [12]. They contain coumarins, flavones, chalcones and meroterpenes, which have antitumor, antibacterial, anti-inflammatory, antimelanogenic and antiosteoporotic activities [13-20]. We previously reported several physiological activities of compounds purified from PC [16,17,19,21,22]. We also found that the major compound of PC, bakuchiol, has potential myogenic activity [23]. In this study, we attempted to identify additional potent myogenic agents that could be derived from PC. Further, we wished to uncover mechanisms by which the myogenic capacity of myoblasts and protective activity of muscle fibers against glucocorticoid-induced damage can be enhanced.

\section{Results}

\subsection{Extracts of Psoralea Corylifolia Enhance Myogenesis}

The myogenic activity of ethanol extracts of Psoralea corylifolia L. (EPC) was evaluated in $\mathrm{C} 2 \mathrm{C} 12$ myoblasts cultures. Mouse $\mathrm{C} 2 \mathrm{C} 12$ cells are derived from muscle satellite cells and can be differentiated into myotubes by cell-cell contact under low serum conditions [10]. Treatment with differentiation medium (DM) triggers differentiation of $\mathrm{C} 2 \mathrm{C} 12$ cells, which then gradually convert into long, tubular myocytes to build muscle bundles.

C2C12 myoblasts were differentiated in DM with the indicated concentrations of EPC $(1,10,100$ and $1000 \mathrm{ng} / \mathrm{mL})$ for three days. They expressed myosin heavy chain (MHC) as a terminal myogenesis marker [10] that can be observed with immunostaining for MHC (red) and 4'-6-diamidino-2-prenylindole (DAPI) (blue). Immunostaining results showed that EPC increased the numbers of cylinder-shaped and multinucleated myotubes in a dose-dependent manner (Figure 1A). Western blot analysis revealed that EPC dose-dependently increased MHC expression of myotubes up to 3.5-fold, as compared with the control (Figure 1B). Pan-cadherin was used as a loading control. 
A
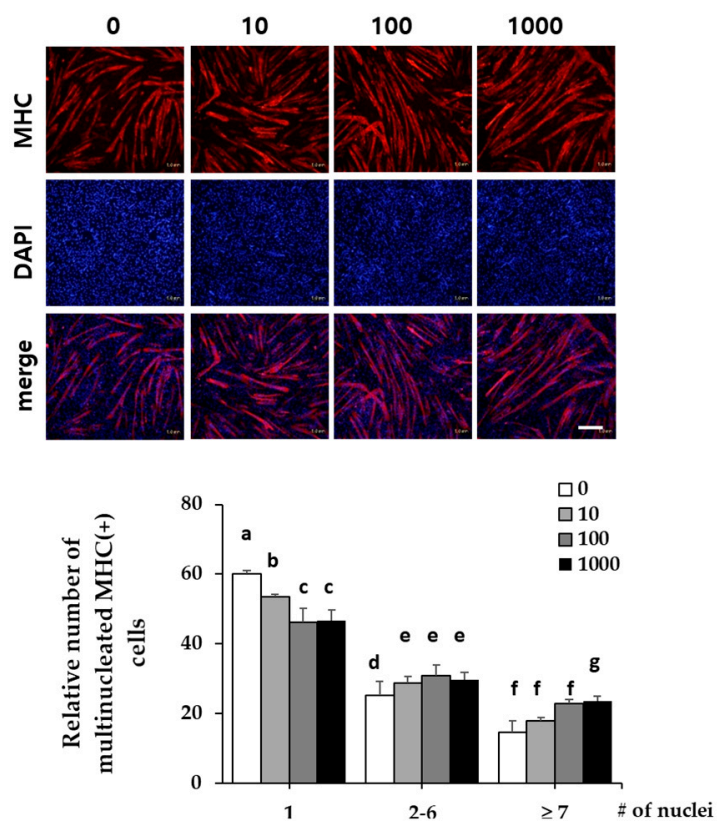

B

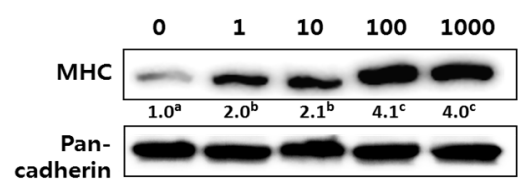

Figure 1. Effect of ethanol extracts of Psoralea corylifolia (EPC) on myogenesis. C2C12 cells were supplemented with differentiation medium (DM), including the indicated concentrations of EPC (1, 10,100 and $1000 \mathrm{ng} / \mathrm{mL}$ ) for 3 days, and then cells were collected to perform (A) immunostaining of MHC (red) and DAPI (blue) (scale bar $=200 \mu \mathrm{m}$ ) and (B) Western blot analysis to determine the level of myosin heavy chain (MHC) expression. The level of MHC protein was quantified and normalized to pan-cadherin. Values are mean $(n=3)$. The images are representative of three independent experiments with similar results. Means without a common superscript differ significantly $(p<0.05)$.

To identify the myogenic compounds from EPC, we purified five flavonoids and three chalcones and identified their structures as being bavachinin (1), isobavachromene (2), bavachalcone (3), corylin (4), bavachin (5), isobavachalcone (6), corylifol A (7) and neobavaisoflavone (8) by spectroscopic data analysis (Figure 2A) [24-28]. We measured endogenous transcriptional activity of MyoD in C2C12 cells to evaluate the myogenic potential of the purified compounds. MyoD is required for terminal specification in muscle cell lineages and functions as a transcriptional factor to induce the expression of myogenic regulatory factors [29]. All of the compounds purified from PC significantly increased MyoD transcriptional activity in myoblasts. Among them, corylifol A (7) was highest and increased MyoD transactivation by 2.3-fold, as compared with the control (Figure 2B). Therefore, we chose corylifol A as the most potent myogenic component from PC and carried out the following experiment to clarify its myogenic potential and underlying mechanisms.

A
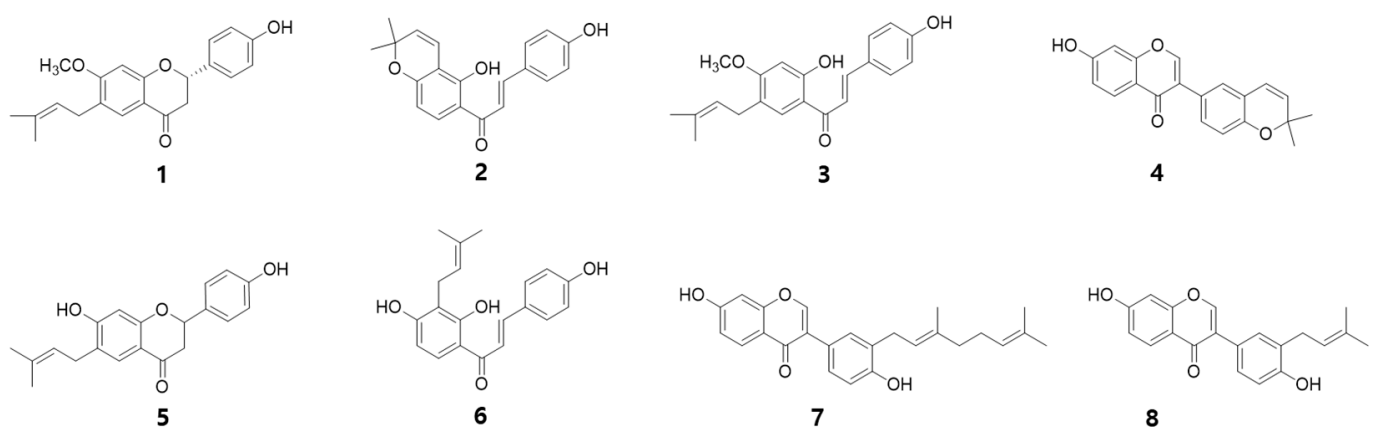

Figure 2. Cont. 


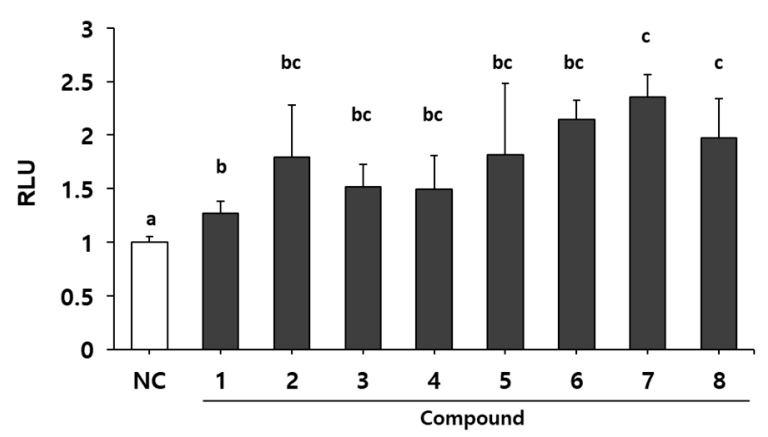

Figure 2. Myogenic effect of compounds 1-8 isolated from PC. (A) Structures of 1-8. (B) Transiently transfected C2C12 cells with a MyoD-responsive reporter gene were differentiated in the presence of each compound (100 nM), followed by measurement of luciferase activity. Data are expressed as mean \pm S.D. of three independent experiments. RLU: relative luciferase units. NC: negative control (DMSO) and 1-8: compounds 1-8. Means without a common superscript differ significantly $(p<0.05)$.

\subsection{Corylifol A Promotes Myogenesis}

Since corylifol A induced the highest MyoD transcriptional activity, we evaluated its myogenic potential in detail. $\mathrm{C} 2 \mathrm{C} 12$ myoblasts were differentiated in the presence of the indicated concentrations $(10,50$ and $100 \mathrm{nM})$ of corylifol A for three days, and then the number of differentiated myotubes were assessed by immunostaining for MHC and DAPI. The MHC-positive cells were scored with respect to the number of nuclei to quantify myoblast differentiation. Corylifol A dose-dependently increased the number of MHC-positive multinucleated myotubes, while it decreased the number of mononuclear myotubes (Figure 3A). Accordingly, corylifol A also dose-dependently enhanced the expression of myogenic factors, such as MHC, myogenin and $\mathrm{MyoD}$ (Figure 3B), and elevated MyoD transactivation (Figure 3C).

To monitor the expression of myogenic factors during the differentiation period, $\mathrm{C} 2 \mathrm{C} 12$ myoblasts were treated with $100 \mathrm{nM}$ corylifol A for the three days of differentiation. As differentiation progressed, the expression of MHC and myogenin gradually increased and reached a maximum on differentiation at day three (D3), while maximal MyoD expression was observed on day two (D2) (Figure 3D). Corylifol A treatment further increased the expressions of MHC, myogenin and MyoD, as compared with their respective day control. Collectively, our results demonstrate that corylifol A enhances myogenesis in dose- $(<100 \mathrm{nM})$ and time-dependent ( $<3$ days) manners through the induction of MyoD expression as the early regulator of myoblast differentiation.

A
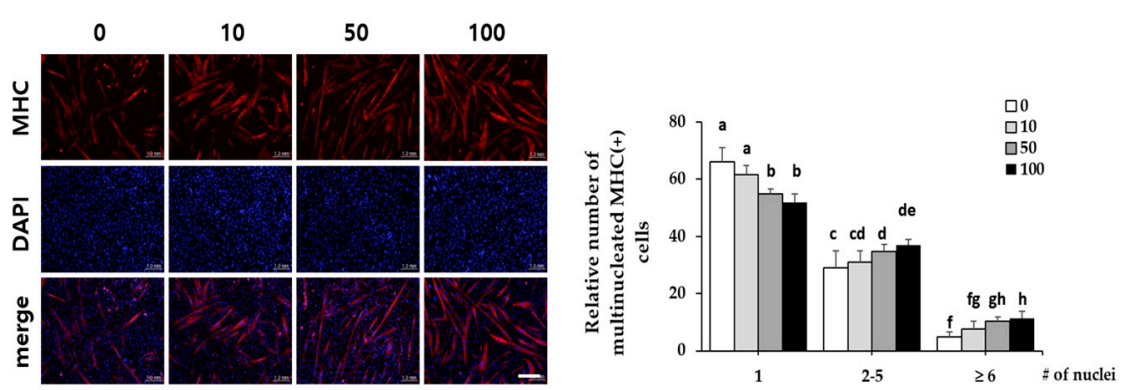

Figure 3. Cont. 
B

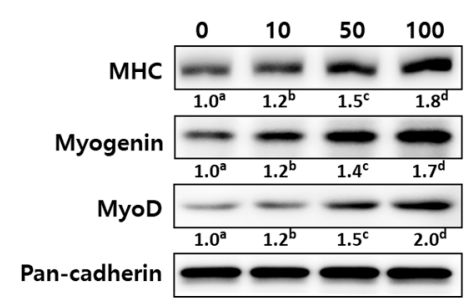

C

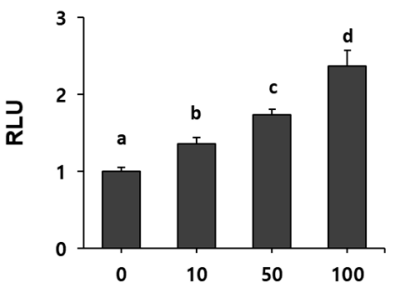

D

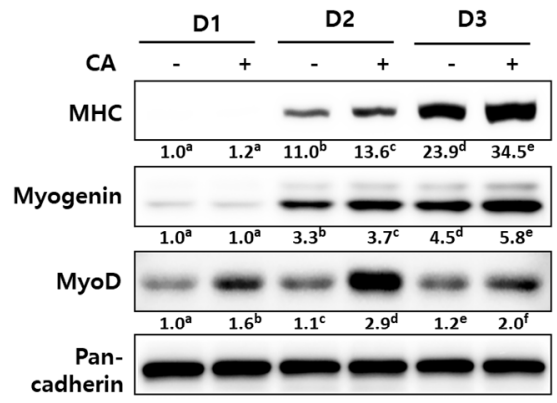

Figure 3. Effect of corylifol A on myogenesis. C2C12 cells were differentiated in the presence of corylifol A (CA, 10, 50 and $100 \mathrm{nM}$ ) for 3 days, and then cells were collected to perform (A) immunostaining of MHC (red) and DAPI (blue) (scale bar $=200 \mu \mathrm{m}$ ) and (B) Western blot analysis to determine the expression of myogenic markers. Values are mean $(n=3)$. (C) C2C12 myoblasts were transfected with a MyoD-responsive reporter 4RTK-luciferase, and then cells were differentiated in the presence of corylifol A, followed by measurement of luciferase activity. Data are expressed as mean \pm S.D. of three independent experiments. (D) C2C12 myoblasts treated with corylifol A (100 nM) and harvested on differentiation day 1 (D1), 2 (D2) and 3 (D3) to evaluate the expression of myogenic markers. Values are mean $(n=3)$. The images are representative of three independent experiments with similar results. Means without a common superscript differ significantly $(p<0.05)$.

\subsection{Corylifol A Promotes Myogenesis via p38 MAPK Activation}

To elucidate the underlying mechanisms of corylifol A for myogenesis, we checked the level of phospho-p38 mitogen-activated protein kinase (p38 MAPK) and phospho-protein kinase B (Akt), as these are known to be regulatory kinases [30,31] during differentiation. As shown in Figure 4A, the level of phosphorylated p38 MAPK was steady for two days after DM treatment and then slowly declined. The addition of corylifol A (100 nM) further sustained p38 MAPK activation, as compared with the corresponding control. In the case of the Akt pathway, phosphorylated Akt levels were maximal on D2 but were not affected by corylifol A during myoblast differentiation, compared with the respective day control.

To confirm the role of p38 MAPK in corylifol A-induced myogenesis, C2C12 myoblasts were pretreated with SB203580, a pyridinyl imidazole inhibitor of p38 MAPK, and then differentiated in the presence of corylifol A. Treatment with SB203580 showed that suppressed expression of myogenic markers could not be restored by corylifol A (Figure 4B). Immunostaining results were consistent with protein expression data for myogenic markers and showed that the number of MHC-positive multinucleated myotubes were decreased by SB203580 and were not restored by corylifol A (Supplementary Figure S1). Taken together, corylifol A induced myogenesis through the activation of the p38 MAPK pathway that is known to be required for the process. 
A

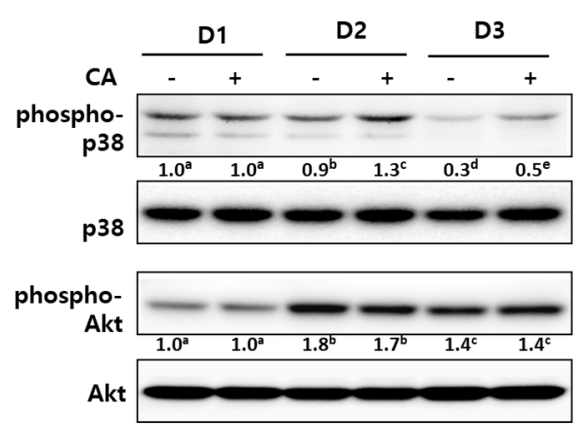

B

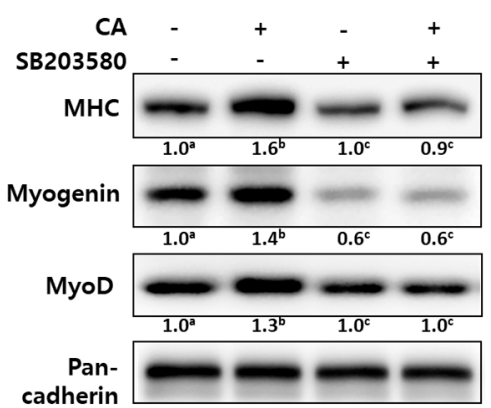

Figure 4. Effect of corylifol A on p38 MAPK activation during myogenesis. (A) C2C12 cells were treated with corylifol A (CA, $100 \mathrm{nM}$ ) during differentiation and subjected to Western blot analysis on differentiation day 1 (D1), 2 (D2) or 3 (D3). The levels of phospho-p38 and Akt were normalized against the respective total protein level. (B) C2C12 cells were pretreated with SB203580 $(10 \mu \mathrm{M})$ prior to corylifol A and then differentiated in DM for 2 days. Cell lysates were subjected to Western blot analysis. Values are mean $(n=3)$. The images are representative of three independent experiments with similar results. Means without a common superscript differ significantly $(p<0.05)$.

\subsection{Corylifol A Protects against Dexamethasone-Induced Muscle Atrophy in Vitro}

To investigate the protective effect of corylifol A against muscle atrophy, fully differentiated myotubes were further incubated with dexamethasone to induce atrophy. A synthetic glucocorticoid, dexamethasone, has been well-known to induce muscle atrophy in vitro and in vivo $[10,32]$. In the inflammatory environment that follows dexamethasone treatment, the activation of the NF- $\mathrm{kB}$ pathway is essential for the expression of myostatin and muscle-specific E3 ligases, such as the muscle atrophy F-box (MAFbx/atrogin-1) and muscle RING finger 1 (MuRF1), to degrade muscle proteins [33].

As expected, dexamethasone treatment $(1 \mu \mathrm{M})$ greatly decreased the number of MHC-expressing multinucleated myotubes, but a 48-h treatment with $100 \mathrm{nM}$ corylifol A protected against myotube loss (Figure 5A). Next, we measured the levels of muscular catabolic and anabolic factors in dexamethasone-damaged myotubes. Dexamethasone activated NF- $\mathrm{kB}$ and increased the expression of myostatin and E3 ligases (catabolic mechanism), as compared with the vehicle control. Cotreatment with dexamethasone and corylifol A diminished their expression, as compared with the dexamethasone control (Figure 5B).

The PI3K/Akt pathway has been shown to be activated by stimuli such as insulin and to be a central anabolic pathway leading to increased skeletal muscle mass and size [34]. As shown in Figure 5B, dexamethasone decreased the expression of MHC and the phosphorylated Akt compared to the control, but corylifol A rescued the expression of these anabolic factors. These data suggested that corylifol A protects against dexamethasone-induced muscle atrophy in vitro by contributing to the inhibition of catabolic pathways and by the activation of the anabolic pathway in muscles. 
A

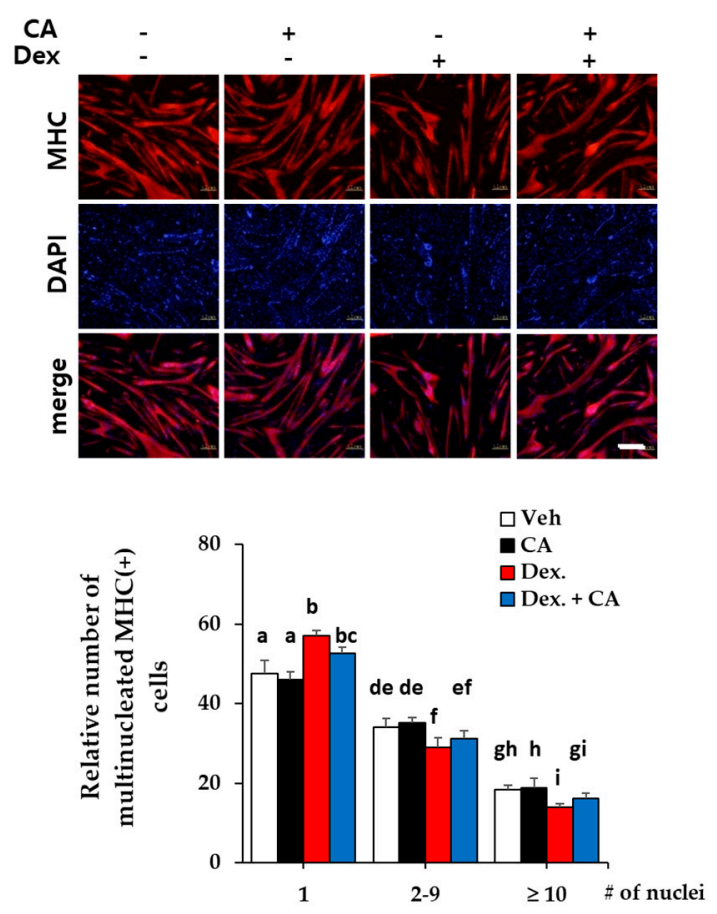

B

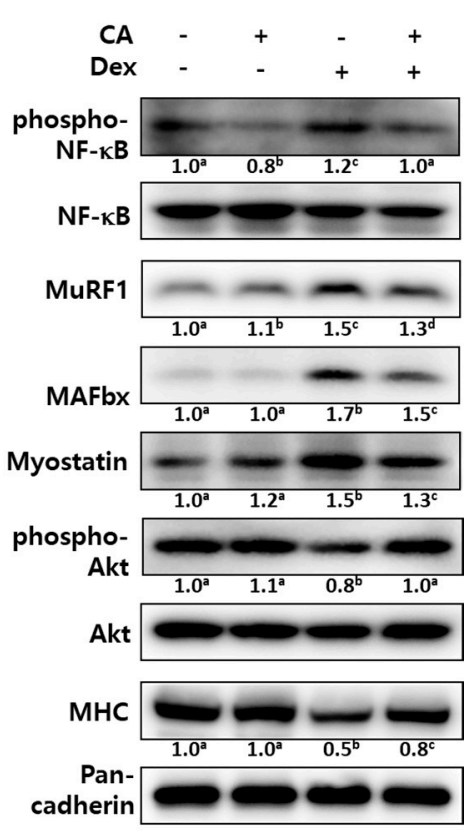

Figure 5. Protective effects of corylifol A against dexamethasone-induced myotube atrophy in vitro. C2C12 myoblasts were differentiated for 3 days, followed by treatment with dexamethasone (Dex, $1 \mu \mathrm{M}$ ) along with vehicle DMSO or corylifol A (CA, $100 \mathrm{nM}$ ) for an additional $48 \mathrm{~h}$. (A) Collected cells were fixed and immunostained with MHC (red) and DAPI (blue) (scale bar $=200 \mu \mathrm{m}$ ). (B) Cell lysates were subjected to Western blot analysis to analyze phosphorylated NF- $\mathrm{kB}$, E3 ligases, myostatin, phosphorylated Akt and MHC. Values are mean $(n=3)$. The images are representative of three independent experiments with similar results. Means without a common superscript differ significantly $(p<0.05)$.

\section{Discussion}

Quiescent muscle satellite cells are activated by various stimuli and undergo myogenesis to form muscle fibers and regenerate skeletal muscle. Myogenesis is a multistage process by which different muscle-specific regulatory factors like MyoD, myogenic regulatory factor (Myf)-5, myogenin and myosin heavy chain $(\mathrm{MHC})$, are expressed at different times during the myoblast differentiation $[29,35,36]$. MyoD and Myf-5 act as initial transcriptional regulators of myogenesis. In particular, MyoD heterodimerizes with $\mathrm{E}$ proteins and cooperates with myocyte enhancer factor (MEF)-2 family transcription factors to express the target gene, myogenin. Subsequently, myogenin induces relevant muscle specific factors like MHC, leading to terminal differentiation [37].

In this study, we purified eight compounds from an ethanol extract of P. corylifolia (EPC) to identify new myogenic agents. We identified corylifol A as a promising component of EPC that stimulated myogenesis in an in vitro experimental model using C2C12 myoblasts. Corylifol A induced the highest MyoD transcriptional activity among the eight PC compounds. It increased MHC-positive multinucleated myotubes together with increased expressions of myogenic factors, such as MHC, myogenin and MyoD (Figures 2 and 3).

At an early stage of myogenesis, p38 mitogen-activated protein kinase (p38 MAPK) mediates the phosphorylation of E proteins to facilitate heterodimerization with MyoD followed by the expression of muscle-specific genes and the prevention of premature myogenesis in activated satellite cells [38,39]. As shown in Figure 4, corylifol A enhanced the phosphorylation level of p38 MAPK on differentiation day 2 (D2) and further sustained the level on D3 to stimulate myogenesis. Another myogenic kinase, Akt was not affected by corylifol A. 
To mimic muscle atrophy in vitro, differentiated myotubes were treated with dexamethasone, a synthetic glucocorticoid, for two days. Dexamethasone decreased the number of MHC-expressing multinucleated myotubes, which is a hallmark of muscle atrophy in vitro [10]. Corylifol A also protected against the impaired myotube formations, as compared with dexamethasone control (Figure 5A). As another strategy to overcome muscle atrophy, the regulation of muscle protein metabolism has been considered [6]. In a muscle atrophic environment, NF-KB-mediated E ubiquitin ligases (MuRF1 and $\mathrm{MAFbx}$ ) are responsible for proteasomal degradation of muscle protein. Myostatin is a major negative regulator of skeletal muscle mass by acting on muscle myokine to inhibit myogenesis. As excessive myostatin accelerates muscle atrophy, inhibitors of myostatin have been attracting attention as potential targets for muscle atrophy therapeutics [6,40]. Dexamethasone increased expression levels of NF-kB-mediated E3 ligases and myostatin, indicating muscle atrophy in vitro. Corylifol A suppressed the expression of phosphorylated NF- $\mathrm{kB}, \mathrm{MuRF} 1, \mathrm{MAFbx}$ and myostatin in myotubes, as compared with dexamethasone alone (Figure 5B). On the other hand, the PI3K/protein kinase B (Akt) pathway has been considered to be not only involved in the process of skeletal muscle synthesis but also required for cell survival during myogenesis. The decreased phosphorylation of Akt by dexamethasone treatment was restored by corylifol A, accompanied by increased expression of MHC.

Several flavones exert beneficial effects on muscle maintenance. Apigenin, abundant in parsley and celery, was reported to promote myogenesis and prevent muscle weakness via Prmt7-PGC-1 $\alpha$-GPR56 and Prmt7-p38-myoD pathways [41]. Luteolin also prevents lipopolysaccharide-induced muscle atrophy, partly through the regulation of MAFbx expression in vitro [7]. Resveratrol and isoflavones such as genistein and daidzein are also known to have the protective effect against muscle atrophy [42,43]. Despite numerous attempts to discover agents that can inhibit proteasomal degradation and activate protein synthesis of skeletal muscle, further investigations are ongoing for their therapeutic applications.

Corylifol A, an isoflavone purified from P. corylifolia, has diverse physiological activities, including phytoestrogenic [44], antioxidative [18], antihepatoma [45] and hepatoprotective activities [46]. In an earlier study, we isolated a meroterpene compound, bakuchiol, a primary component of P. corylifolia (PC), and disclosed its myogenic potential in $\mathrm{C} 2 \mathrm{C} 12$ myoblasts [23]. Here, we evaluated the myogenic activity of flavonoids and chalcones isolated from PC and identified corylifol A as the most effective ingredient. Corylifol A activates the differentiation of myoblasts and suppresses muscle degradation in dexamethasone-induced muscle atrophic conditions.

\section{Materials and Methods}

\subsection{Preparation of the Ethanol Extracts of P. corylifolia L. (PC) and Purification of Flavonoids and Chalcones}

PC seeds were purchased from the Jinheung Herbal Medicine Market (Seoul, Korea) and deposited as voucher specimen (No. SPH-13003) in the Herbarium of Sookmyung Women's University. The dried and powdered seeds were extracted with ethanol at room temperature for $24 \mathrm{~h}$, and the ethanol extract was partitioned with water and ethyl acetate. The eight compounds were isolated from ethyl acetate soluble fractions, as reported previously $[16,17,21]$. Their structures were elucidated by the analysis of infrared spectroscopy, mass and NMR spectroscopic data, as follows [13,18], bavachinin (1), isobavachromene (2), bavachalcone (3), corylin (4), bavachin (5), isobavachalcone (6), corylifol A (7) and neobavaisoflavone (8).

\subsection{Cell Culture}

C2C12 myoblasts (American Type Culture Collection, Manassas, VA, USA) were maintained in growth medium (GM) composed of Dulbecco's Modified Eagle's Medium (DMEM) containing 15\% fetal bovine serum (Gibco BRL Life Technology, Grand island, NY, USA); $100 \mathrm{U} / \mathrm{mL}$ penicillin and 100 $\mu \mathrm{g} / \mathrm{mL}$ streptomycin (Life Technologies, Frederick, MD, USA). When cells reached $95 \%$ confluence, differentiation of myoblasts was initiated by supplementation with differentiation medium (DM) composed of DMEM containing $2 \%$ horse serum (differentiation day 0: D0). At 3 days post-DM 
treatment (D3), cells were subjected to analytical experiments and image analysis. To investigate potential effects of corylifol A on muscle atrophy, differentiated myotubes were treated with corylifol A $(100 \mathrm{nM})$ for $3 \mathrm{~h}$ prior to dexamethasone ( $1 \mu \mathrm{M}$; Sigma-Aldrich, St. Louis, MO, USA) treatment.

\subsection{Immunostaining of $M H C$}

C2C12 myoblasts and differentiated myotubes were fixed with $4 \%$ paraformaldehyde for $20 \mathrm{~min}$ and permeabilized with $0.1 \%$ Triton X-100 for $20 \mathrm{~min}$ in phosphate-buffered saline (PBS). After washing with PBS, cells were stained with antibody against myosin heavy chain (MHC) (MAB4470; R\&D Systems, Minneapolis, MN, USA) overnight at $4{ }^{\circ} \mathrm{C}$, followed by an Alexa Fluor 568-conjugated secondary antibody (Life Technologies, Carlsbad, CA, USA). Nuclei were counterstained with 4'-6-diamidino-2-prenylindole (Sigma-Aldrich, St. Louis, MO, USA). Immunofluorescence was observed and captured using a fluorescence microscope (Olympus, Tokyo, Japan). Red fluorescence indicates MHC expression, and blue fluorescence indicates nuclei. The MHC-positive cells were grouped according to their number of nuclei in several fields and then presented as the relative cell counts of each group.

\subsection{MyoD-Reporter Gene Assay}

To investigate the transcriptional activity of $\mathrm{MyoD}, \mathrm{C} 2 \mathrm{C} 12$ myoblasts were seeded in 24-well plates at a density of $2 \times 10^{4}$ cells per well. Cells were transiently transfected with MyoD-responsive reporter 4RTK-luciferase (RTK-luc) and pBP-MyoD constructs [10] using lipofectamine LTX (Invitrogen, Calsbad, CA, USA) and incubated for $24 \mathrm{~h}$. The transfected cells were treated with test samples for $48 \mathrm{~h}$. Cell lysates were subjected to luciferase activity assay using a luciferase assay kit (Promega, Madison, WI, USA). Results are presented as relative luciferase activity divided by $\beta$-galactosidase activity.

\subsection{Western Blot Analysis}

To measure the expression of myogenic markers and E3 ligases, cells were lysed in lysis buffer (50 mM Tris, pH 7.4, $150 \mathrm{mM} \mathrm{NaCl}, 10 \%$ glycerol, $1.5 \mathrm{mM} \mathrm{MgCl}_{2}, 1 \mathrm{mM}$ EGTA, $1 \%$ Triton X-100, $10 \mathrm{mM} \mathrm{NaF}, 1 \mathrm{mM} \mathrm{Na}_{3} \mathrm{VO}_{4}$, protease inhibitor cocktail (cOmplete, Roche Applied Science, Mannheim, Germany) and phosphatase inhibitor cocktail (PhosSTOP, Roche) [47]). Whole cell extracts were subjected to sodium dodecyl sulfate-polyacrylamide gel electrophoresis (SDS-PAGE), and the separated proteins were transferred onto a polyvinylfluoride (PVDF) membrane. The membrane was incubated with primary antibodies against myosin heavy chain (MHC), MyoD, myogenin, myostatin, MuRF1 (all Santa Cruz Biotechnology, Inc., Dallas, TX, USA), p38 MAPK, phospho-p38 MAPK, Akt, phospho-Akt, NF-kB, phospho-NF- $\mathrm{B}$ (all Cell Signaling Technology, Inc., Beverly, MA, USA), pan-cadherin and $\beta$-actin (both Sigma-Aldrich). The protein levels were quantified using the Fusion Solo system (Vilber Lourmat, Collegien, France).

\subsection{Statistical Analysis}

The experiments were performed at least three times. Data are expressed as mean \pm standard deviation, and differences between values were assessed using one-way analysis of variance (ANOVA) followed by Duncan's test. ${ }^{*} p<0.05$ was considered statistically significant.

\section{Conclusions}

In summary, the ethanol extract of P. corylifolia (EPC) enhances myoblast differentiation. As the most potent component of EPC, corylifol A showed beneficial and counteracting effects on muscle atrophy via the stimulation of myogenesis and regulation of muscle protein metabolism. Corylifol A stimulates myogenesis by activating the p38 MAPK pathway and ameliorates dexamethasone-induced muscle atrophy by suppressing the NF-kB-mediated E3 ligase machinery (catabolic) and activating 
Akt (anabolic). This dual effect of corylifol A will provide insights into new strategies for overcoming muscle atrophy.

Supplementary Materials: Supplementary materials can be found at http://www.mdpi.com/1422-0067/21/5/1571/s1. Figure S1: Effect of corylifol A on p38 MAPK activation during myogenesis.

Author Contributions: Biological experiments, Y.H.; preparation of extracts and isolation of compounds, Hua Li; preparation of original draft, Hyejin Lee and draft revision and supervision of project, J-H.R. All authors have read and agreed to the published version of the manuscript.

Funding: This research was supported by the Sookmyung Women's University Specialization Program Funding (1-201809-6).

Conflicts of Interest: The authors declare no conflict of interest.

\section{References}

1. Kalyani, R.R.; Corriere, M.; Ferrucci, L. Age-related and disease-related muscle loss: the effect of diabetes, obesity, and other diseases. Lancet Diabetes Endocrinol. 2014, 2, 819-929. [CrossRef]

2. Sakuma, K.; Yamaguchi, A. Sarcopenia and age-related endocrine function. Int. J. Endocrinol. 2012, 2012, 127362. [CrossRef] [PubMed]

3. Welle, S. Cellular and molecular basis of age-related sarcopenia. Can. J. Appl. Physiol. 2002, 27, 19-41. [CrossRef] [PubMed]

4. Cohen, S.; Nathan, J.A.; Goldberg, A.L. Muscle wasting in disease: molecular mechanisms and promising therapies. Nat. Rev. Drug Discov. 2015, 14, 58-74. [CrossRef] [PubMed]

5. Dumont, N.A.; Wang, Y.X.; Rudnicki, M.A. Intrinsic and extrinsic mechanisms regulating satellite cell function. Dev. (Camb. Engl.) 2015, 142, 1572-1581. [CrossRef] [PubMed]

6. Dutt, V.; Gupta, S.; Dabur, R.; Injeti, E.; Mittal, A. Skeletal muscle atrophy: Potential therapeutic agents and their mechanisms of action. Pharmacol. Res. 2015, 99, 86-100. [CrossRef]

7. Mazibuko-Mbeje, S.E.; Dludla, P.V.; Nkambule, B.B.; Obonye, N.; Louw, J. Skeletal muscle as a therapeutic target for natural products to reverse metabolic syndrome. Muscle Cell Tissue: Curr. Status Res. Field 2018, 175. [CrossRef]

8. Lee, H.; Heo, J.W.; Kim, A.R.; Kweon, M.; Nam, S.; Lim, J.S.; Sung, M.K.; Kim, S.E.; Ryu, J.H. Z-ajoene from cushed garlic alleviates cancer-induced skeletal muscle atrophy. Nutrients 2019, 11, 2724. [CrossRef] [PubMed]

9. Kweon, M.; Lee, H.; Park, C.; Choi, Y.H.; Ryu, J.H. A Chalcone from Ashitaba (Angelica keiskei) stimulates myoblast differentiation and inhibits dexamethasone-induced muscle atrophy. Nutrients 2019, 11, 2419. [CrossRef]

10. Lee, H.; Lee, S.J.; Bae, G.U.; Baek, N.I.; Ryu, J.H. Canadine from Corydalis turtschaninovii stimulates myoblast differentiation and protects against myotube atrophy. Int. J Mol. Sci. 2017, 18, 2748. [CrossRef]

11. Hwang, J.; Lee, S.J.; Yoo, M.; Go, G.Y.; Lee, D.Y.; Kim, Y.K.; Seo, D.W.; Kang, J.S.; Ryu, J.H.; Bae, G.U. Kazinol-P from Broussonetia kazinoki enhances skeletal muscle differentiation via p38MAPK and MyoD. Biochem. Biophys. Res. Commun. 2015, 456, 471-475. [CrossRef] [PubMed]

12. Alam, F.; Khan, G.N.; Asad, M. Psoralea corylifolia L: Ethnobotanical, biological, and chemical aspects: A review. Phytother. Res. 2018, 32, 597-615. [CrossRef] [PubMed]

13. Choi, Y.H.; Yon, G.H.; Hong, K.S.; Yoo, D.S.; Choi, C.W.; Park, W.K.; Kong, J.Y.; Kim, Y.S.; Ryu, S.Y. In vitro BACE-1 inhibitory phenolic components from the seeds of Psoralea corylifolia. Planta Med. 2008, 74, 1405-1408. [CrossRef] [PubMed]

14. Cui, Y.; Taniguchi, S.; Kuroda, T.; Hatano, T. Constituents of Psoralea corylifolia fruits and their effects on methicillin-resistant staphylococcus aureus. Molecules 2015, 20, 12500-12511. [CrossRef] [PubMed]

15. Don, M.J.; Lin, L.C.; Chiou, W.F. Neobavaisoflavone stimulates osteogenesis via p38-mediated up-regulation of transcription factors and osteoid genes expression in MC3T3-E1 cells. Phytomedicine 2012, 19, 551-561.

16. Kim, D.H.; Li, H.; Han, Y.E.; Jeong, J.H.; Lee, H.J.; Ryu, J.H. Modulation of inducible nitric oxide synthase expression in LPS-Stimulated BV-2 microglia by prenylated chalcones from Cullen corylifolium (L.) Medik. through inhibition of I-kB $\alpha$ degradation. Molecules 2018, 23, 109. 
17. Lee, M.H.; Kim, J.Y.; Ryu, J.H. Prenylflavones from Psoralea corylifolia inhibit nitric oxide synthase expression through the inhibition of I-kB- $\alpha$ degradation in activated microglial cells. Biol. Pharm. Bull. 2005, 28, 2253-2257. [CrossRef]

18. Lee, S.W.; Yun, B.R.; Kim, M.H.; Park, C.S.; Lee, W.S.; Oh, H.M.; Rho, M.C. Phenolic compounds isolated from Psoralea corylifolia inhibit IL-6-induced STAT3 activation. Planta Med. 2012, 78, 903-906. [CrossRef]

19. Lim, J.; Nam, S.; Li, H.; Yang, Y.; Lee, M.S.; Lee, H.G.; Ryu, J.H.; Lim, J.S. Antimelanogenic effect of 4-hydroxylonchocarpin through the inhibition of tyrosinase-related proteins and MAPK phosphatase. Exp. Dermatol. 2016, 25, 574-576. [CrossRef]

20. Tsai, M.H.; Huang, G.S.; Hung, Y.C.; Bin, L.; Liao, L.T.; Lin, L.W. Psoralea corylifolia extract ameliorates experimental osteoporosis in ovariectomized rats. Am. J. Chin. Med. 2007, 35, 669-680. [CrossRef]

21. Lee, H.; Li, H.; Noh, M.; Ryu, J.H. Bavachin from Psoralea corylifolia improves insulin-dependent glucose uptake through insulin signaling and AMPK activation in 3T3-L1 adipocytes. Int. J. Mol. Sci. 2016, 17, 527. [CrossRef] [PubMed]

22. Park, C.K.; Lee, Y.; Chang, E.J.; Lee, M.H.; Yoon, J.H.; Ryu, J.H.; Kim, H.H. Bavachalcone inhibits osteoclast differentiation through suppression of NFATc1 induction by RANKL. Biochem. Pharmacol. 2008, 75, 2175-2182. [CrossRef]

23. Lee, S.J.; Yoo, M.; Go, G.Y.; Kim do, H.; Choi, H.; Leem, Y.E.; Kim, Y.K.; Seo, D.W.; Ryu, J.H.; Kang, J.S.; et al. Bakuchiol augments MyoD activation leading to enhanced myoblast differentiation. Chem. Biol. Int. 2016, 248, 60-67. [CrossRef] [PubMed]

24. Wang, D.; Li, F.; Jiang, Z. Osteoblastic proliferation stimulating activity of Psoralea corylifolia extracts and two of its flavonoids. Planta Med. 2001, 67, 748-749. [CrossRef] [PubMed]

25. Chen, Q.; Li, Y.; Chen, Z. Separation, identification, and quantification of active constituents in Fructus Psoraleae by high-performance liquid chromatography with UV, ion trap mass spectrometry, and electrochemical detection. J. Pharm. Anal. 2012, 2, 143-151. [CrossRef] [PubMed]

26. Pistelli, L.; Spera, K.; Flamini, G.; Mele, S.; Morelli, I. Isoflavonoids and chalcones from Anthyllis hermanniae. Phytochem. 1996, 42, 1455-1458. [CrossRef]

27. Yin, S.; Fan, C.Q.; Wang, Y.; Dong, L.; Yue, J.M. Antibacterial prenylflavone derivatives from Psoralea corylifolia, and their structure-activity relationship study. Bioorg. Med. Chem. 2004, 12, 4387-4392. [CrossRef]

28. Nicolaou, K.C.; Cao, G.Q.; Pfefferkorn, J.A. Selenium-based solid-phase synthesis of benzopyrans II: Applications to combinatorial synthesis of medicinally relevant small organic molecules. Angew. Chem. Int. Ed. Engl. 2000, 39, 739-743. [CrossRef]

29. Bentzinger, C.F.; Wang, Y.X.; Rudnicki, M.A. Building muscle: molecular regulation of myogenesis. Cold Spring Harb Perspect Biol. 2012, 4, a008342. [CrossRef]

30. Lassar, A.B. The p38 MAPK family, a pushmi-pullyu of skeletal muscle differentiation. J. Cell. Biol. 2009, 187, 941-943. [CrossRef]

31. Vandromme, M.; Rochat, A.; Meier, R.; Carnac, G.; Besser, D.; Hemmings, B.A.; Fernandez, A.; Lamb, N.J. Protein kinase B beta/Akt2 plays a specific role in muscle differentiation. J. Biol. Chem. 2001, 276, 8173-8179. [CrossRef]

32. Kim, J.W.; Ku, S.K.; Han, M.H.; Kim, K.Y.; Kim, S.G.; Kim, G.Y.; Hwang, H.J.; Kim, B.W.; Kim, C.M.; Choi, Y.H. The administration of Fructus Schisandrae attenuates dexamethasone-induced muscle atrophy in mice. Int. J. Mol. Med. 2015, 36, 29-42. [CrossRef]

33. Menconi, M.; Gonnella, P.; Petkova, V.; Lecker, S.; Hasselgren, P.O. Dexamethasone and corticosterone induce similar, but not identical, muscle wasting responses in cultured L6 and C2C12 myotubes. J. Cell Biochem. 2008, 105, 353-364. [CrossRef]

34. Jaiswal, N.; Gavin, M.G.; Quinn, W.J., 3rd; Luongo, T.S.; Gelfer, R.G.; Baur, J.A.; Titchenell, P.M. The role of skeletal muscle Akt in the regulation of muscle mass and glucose homeostasis. Mol. Metabol. 2019, 28, 1-13.

35. Jin, W.; Peng, J.; Jiang, S. The epigenetic regulation of embryonic myogenesis and adult muscle regeneration by histone methylation modification. Biochem. Biophys. Rep. 2016, 6, 209-219. [CrossRef]

36. Saccone, V.; Puri, P.L. Epigenetic regulation of skeletal myogenesis. Organogenesis 2010, 6, 48-53. [CrossRef]

37. Peng, X.L.; So, K.K.; He, L.; Zhao, Y.; Zhou, J.; Li, Y.; Yao, M.; Xu, B.; Zhang, S.; Yao, H.; et al. MyoD- and FoxO3-mediated hotspot interaction orchestrates super-enhancer activity during myogenic differentiation. Nucleic acids Res. 2017, 45, 8785-8805. [CrossRef] 
38. Bennett, A.M.; Tonks, N.K. Regulation of distinct stages of skeletal muscle differentiation by mitogen-activated protein kinases. Science 1997, 278, 1288-1291. [CrossRef]

39. Forcales, S.V.; Albini, S.; Giordani, L.; Malecova, B.; Cignolo, L.; Chernov, A.; Coutinho, P.; Saccone, V.; Consalvi, S.; Williams, R.; et al. Signal-dependent incorporation of MyoD-BAF60c into Brg1-based SWI/SNF chromatin-remodelling complex. Embo J. 2012, 31, 301-316. [CrossRef]

40. Hoogaars, W.M.H.; Jaspers, R.T. Past, Present, and future perspective of targeting myostatin and related signaling pathways to counteract muscle atrophy. Adv. Exp. Med. Biol. 2018, 1088, 153-206.

41. Jang, Y.J.; Son, H.J.; Choi, Y.M.; Ahn, J.; Jung, C.H.; Ha, T.Y. Apigenin enhances skeletal muscle hypertrophy and myoblast differentiation by regulating Prmt7. Oncotarget 2017, 8, 78300-78311. [CrossRef]

42. Hirasaka, K.; Maeda, T.; Ikeda, C.; Haruna, M.; Kohno, S.; Abe, T.; Ochi, A.; Mukai, R.; Oarada, M.; Eshima-Kondo, S.; et al. Isoflavones derived from soy beans prevent MuRF1-mediated muscle atrophy in C2C12 myotubes through SIRT1 activation. J. Nutr. Sci. Vitaminol. 2013, 59, 317-324. [CrossRef]

43. Murillo Ortiz, B.O.; Fuentes Preciado, A.R.; Ramirez Emiliano, J.; Martinez Garza, S.; Ramos Rodriguez, E.; de Alba Macias, L.A. Recovery of bone and muscle mass in patients with chronic kidney disease and iron overload on hemodialysis and taking combined supplementation with curcumin and resveratrol. Clin. Interv. Aging 2019, 14, 2055-2062. [CrossRef]

44. Xin, D.; Wang, H.; Yang, J.; Su, Y.F.; Fan, G.W.; Wang, Y.F.; Zhu, Y.; Gao, X.M. Phytoestrogens from Psoralea corylifolia reveal estrogen receptor-subtype selectivity. Phytomedicine 2010, 17, 126-131. [CrossRef]

45. Song, P.; Yang, X.Z.; Yuan, J.Q. Cytotoxic constituents from Psoralea corylifolia. J. Asian Nat. Prod. Res. 2013, 15, 624-630. [CrossRef]

46. Wang, X.X.; Lv, X.; Li, S.Y.; Hou, J.; Ning, J.; Wang, J.Y.; Cao, Y.F.; Ge, G.B.; Guo, B.; Yang, L. Identification and characterization of naturally occurring inhibitors against UDP-glucuronosyltransferase 1A1 in Fructus Psoraleae (Bu-gu-zhi). Toxicol. Appl. Pharmacol. 2015, 289, 70-78. [CrossRef] [PubMed]

47. Kurgan, N.; Whitley, K.C.; Maddalena, L.A.; Moradi, F.; Stoikos, J.; Hamstra, S.I.; Rubie, E.A.; Kumar, M.; Roy, B.D.; Woodgett, J.R.; et al. A low-therapeutic dose of lithium inhibits GSK3 and enhances myoblast fusion in C2C12 cells. Cells 2019, 8, 1340. [CrossRef] [PubMed] 